ARTICLE

\title{
Optical focusing inside scattering media with time-reversed ultrasound microbubble encoded light
}

\author{
Haowen Ruan ${ }^{1, \star}$, Mooseok Jang ${ }^{1, \star} \&$ Changhuei Yang ${ }^{1}$
}

Focusing light inside scattering media in a freely addressable fashion is challenging, as the wavefront of the scattered light is highly disordered. Recently developed ultrasound-guided wavefront shaping methods are addressing this challenge, albeit with relatively low modulation efficiency and resolution limitations. In this paper, we present a new technique, time-reversed ultrasound microbubble encoded (TRUME) optical focusing, which can focus light with improved efficiency and sub-ultrasound wavelength resolution. This method ultrasonically destroys microbubbles, and measures the wavefront change to compute and render a suitable time-reversed wavefront solution for focusing. We demonstrate that the TRUME technique can create an optical focus at the site of bubble destruction with a size of $\sim 2 \mu \mathrm{m}$. We further demonstrate a twofold enhancement in addressable focus resolution in a microbubble aggregate target by exploiting the nonlinear pressure-to-destruction response of the microbubbles. The reported technique provides a deep tissue-focusing solution with high efficiency, resolution, and specificity.

\footnotetext{
${ }^{1}$ Department of Electrical Engineering, California Institute of Technology, 1200 East California Boulevard, Pasadena, California 91125, USA. * These authors contributed equally to this work. Correspondence and requests for materials should be addressed to H.R. (email: hruan@caltech.edu) or to M.J. (email: mjang@caltech.edu).
} 
C reating an optical focus inside a scattering medium such as biological tissue has great potential in various applications. However, optical scattering, a dominant light-matter interaction within biological tissue, poses a very significant challenge. Recent developed wavefront shaping techniques have begun to address this ${ }^{1-5}$ by exploiting the deterministic and timesymmetric nature of scattering. Focusing light through scattering media has been realized by iterative optimization methods 2,6 , optical phase conjugation $(\mathrm{OPC})^{7-12}$, and direct measurement of the transmission matrix at large scale ${ }^{13-15}$.

Determining the correct wavefront to focus light from outside of a scattering medium to a point within requires a feedback or tagging mechanism. Typically, these mechanisms arise from a localized 'guidestar' point. Examples of guidestars include second harmonic generation $^{16}$, fluorescence ${ }^{17,18}$ and kinetic ${ }^{19,20}$ targets. While individual guidestars enable light shaped to focus to their physical location, these techniques fundamentally lack addressability if dense and randomly distributed sets of guidestars are present.

Alternatively, ultrasound-assisted techniques, such as photoacoustic-guided ${ }^{15,21-23}$ and time-reversed ultrasonically encoded (TRUE) $^{24-27}$ optical focusing techniques, employ a focused ultrasound beam as a 'virtual guidestar'. Unlike the above techniques, it is easy to move or scan an ultrasound focus to new positions. While TRUE has a speed advantage over the photoacoustic approach, the TRUE guidestar is generally weak and typically $<1 \%$ of the probe light field that passes through the ultrasound focus is tagged ${ }^{28,29}$. Moreover, the resolution achieved is limited by the ultrasound focus size. Although more advanced TRUE techniques, such as iterative TRUE ${ }^{30-32}$ and time reversal of variance-encoded light ${ }^{33}$, may break this resolution barrier, it comes at the expense of time. For practical biological applications with tight time constraints, efficient and fast techniques are highly desired.

Here we present a high resolution, deep tissue optical focusing technique termed time-reversed ultrasound microbubble encoded (TRUME) optical focusing. Microbubbles have been widely used in ultrasonic imaging as ultrasound contrast agents because they generate stronger echoes and nonlinear acoustic signals compared with surrounding tissue ${ }^{34,35}$. Several other advantages of microbubbles are their small size compared with typical ultrasound wavelengths, which enables acoustic super-resolution imaging $^{36-38}$ and their ability to enable ultrasound modulated optical imaging inside scattering media ${ }^{39-41}$. Furthermore, like fluorescent markers, microbubbles can be modified to bind to selected biomarkers, suggesting promise for functional imaging and therapeutic applications ${ }^{34}$.

We demonstrate that the selective nonlinear destruction of microbubbles with a focused ultrasound beam can serve as effective, highly localized and freely addressable guidestar mechanism. In brief, TRUME works by measuring the scattered optical field before and after the ultrasonic destruction of the microbubble. Subsequently, by playing back the phase conjugate version of the difference of these two fields, TRUME can generate a focus at the location of the destroyed microbubble. Although multiple foci could be created at the same time when multiple microbubbles are present within the original ultrasound focus, we show that careful selection of the ultrasound pressure can lead to destruction of microbubbles in an addressable volume that is smaller than the ultrasound focus. This is a result of the nonlinear pressure-to-destruction response curve associated with the microbubbles. This technique combines the advantages of both physical and virtual guidestars to provide efficient, fast and addressable deep tissue optical focusing.

\section{Results}

Principles. Our TRUME set-up uses a digital OPC (DOPC) system as its wavefront recording and playback engine ${ }^{8,31}$
(Fig. 1a). In the recording phase, the scattered field from the sample is recorded by the camera of the DOPC system. In the playback step, a phase-conjugated version of the recorded phase is displayed on the spatial light modulator (SLM) and a collimated 'blank' beam is modulated to form the playback light field. Precise alignment of the camera and SLM allows high fidelity phase conjugate playback of the record field. Experimentally, this DOPC system is able to control $\sim 10^{5}$ optical modes simultaneously ${ }^{42}$.

Here we demonstrate TRUME in transmission geometry (Fig. 1a), in which a sample beam transmits through the sample in the $z$ direction and part of the scattered light is measured by the camera on the other side of the sample. An ultrasound beam is focused on the microbubbles embedded between two diffusers through water coupling. TRUME operates in three steps. First, an optical field (Field A) is measured by the camera (Fig. 1b) with phase shifting digital holography ${ }^{43}$. Second (Fig. 1c), ultrasound is applied to destroy the targeted microbubble, immediately followed by the measurement of a second optical field (Field B). The difference of the fields (Fields $\mathbf{A}-\mathbf{B}$ ) is the scattered field solution associated with the microbubble. The DOPC system computes this difference field and plays back a phase conjugate copy. Since the difference field primarily contains only information from the microbubble, the conjugated beam focuses to the position of the destroyed microbubble (Fig. 1d).

TRUME shares the same mathematical framework as guidestar techniques using kinetic objects ${ }^{19,20}$. The optical field on the target plane $\mathbf{E}_{\mathrm{t}}$ can be decomposed into a microbubble diffracted field $\mathbf{E}_{\mathrm{m}}$ and a background field $\mathbf{E}_{\mathrm{b}}$, which describes the field after microbubble destruction: $\mathbf{E}_{\mathrm{t}}=\mathbf{E}_{\mathrm{m}}+\mathbf{E}_{\mathrm{b}}$. Since the camera and SLM contain discrete components, it is convenient to discretize $\mathbf{E}_{\mathrm{m}}$ and $\mathbf{E}_{\mathrm{b}}$ as column vectors with $n$ complex elements, with each element mapping to an optical mode on the two-dimensional target plane. We may then connect this target field to the field on the measurement plane $\mathbf{E}_{\mathrm{t}}^{\prime}$ through a matrix equation: $\mathbf{E}_{\mathrm{t}}^{\prime}=\mathbf{T E}_{\mathrm{t}}=\mathbf{T}\left(\mathbf{E}_{\mathrm{m}}+\mathbf{E}_{\mathrm{b}}\right)$. Here $\mathbf{T}$ is an $m \times n$ matrix describing the scattering medium and $\mathbf{E}_{\mathrm{t}}^{\prime}$ is a column vector of $m$ elements, with each element mapping to an optical mode on the twodimensional measurement plane. Similarly, the field measured after microbubble destruction can be given by $\mathbf{E}_{\mathrm{b}}^{\prime}=\mathbf{T} \mathbf{E}_{\mathrm{b}}$. The difference field on the measurement plane is thus,

$$
\begin{aligned}
\mathbf{E}_{\mathrm{d}}^{\prime} & =\mathbf{E}_{\mathrm{t}}^{\prime}-\mathbf{E}_{\mathrm{b}}^{\prime} \\
& =\mathbf{T}\left(\mathbf{E}_{\mathrm{m}}+\mathbf{E}_{\mathrm{b}}\right)-\mathbf{T E}_{\mathrm{b}} \\
& =\mathbf{T} \mathbf{E}_{\mathrm{m}} .
\end{aligned}
$$

Here subtraction effectively removes the impact of the background field on the measurement plane, resulting in a field that appears to be scattered from the microbubbles only. Finally, we playback the conjugated field $\mathbf{E}_{\mathrm{d}}^{*}$ with an optical gain $\alpha$ provided by the playback beam (Fig. 1a). Assuming time-reversal symmetry, we may express playback as a multiplication with $\mathbf{T}$ from the left with the conjugate transpose of the difference field. Therefore, the playback field $\mathbf{E}_{\mathrm{p}}$ on the target plane takes the form:

$$
\begin{aligned}
\mathbf{E}_{\mathrm{p}} & =\alpha \mathbf{E}_{\mathrm{d}}^{*} \mathbf{T}=\alpha\left(\mathbf{T E}_{\mathrm{m}}\right)^{*} \mathbf{T} \\
& =\alpha \mathbf{E}_{\mathrm{m}}^{*} \mathbf{T}^{*} \mathbf{T} \approx \alpha \beta \mathbf{E}_{\mathrm{m}}^{*} .
\end{aligned}
$$

Here we assume minimal absorption within the sample to apply the approximation $\mathbf{T}^{*} \mathbf{T} \approx \beta \mathbf{I}$, in which $\beta$ is the fraction of scattered light field that is measured in the DOPC system and I is an identity matrix. The playback light effectively cancels out the random transmission matrix to refocus at the location of microbubble destruction.

The TRUME technique relies on a novel guidestar mechanism, popping a gas-filled microbubble using ultrasound, to generate 
the optical field difference. This mechanism leverages both the optical and acoustic refractive index mismatch between gas and liquid to accomplish efficient conversion of acoustic signal to optical signal. Given the fact that microbubbles have excellent biocompatibility, this guidestar combines its advantages in optics, acoustics and biology to provide a solution for focusing light inside biological tissue.

Visualization and efficiency characterization of the focus. To validate TRUME focusing, we directly visualized the target plane using a $\times 10$ microscope system (see Methods section) before and after the TRUME procedure. In this experiment, we shifted the front diffuser along the $x$ direction (to the 'open position' in Fig. 2a) for direct imaging of the target plane during the focusing phase. The target sample here is composed of microbubbles embedded in agarose gel within an acrylic capillary tube (see Methods section) as shown in Fig. 2b. Immediately after measuring the first optical field, a $20-\mathrm{MHz}$ focused ultrasound beam was used to destroy one microbubble, followed by the measurement of the second field. We then imaged the target plane again to confirm the destruction of the microbubble (Fig. 2c) and

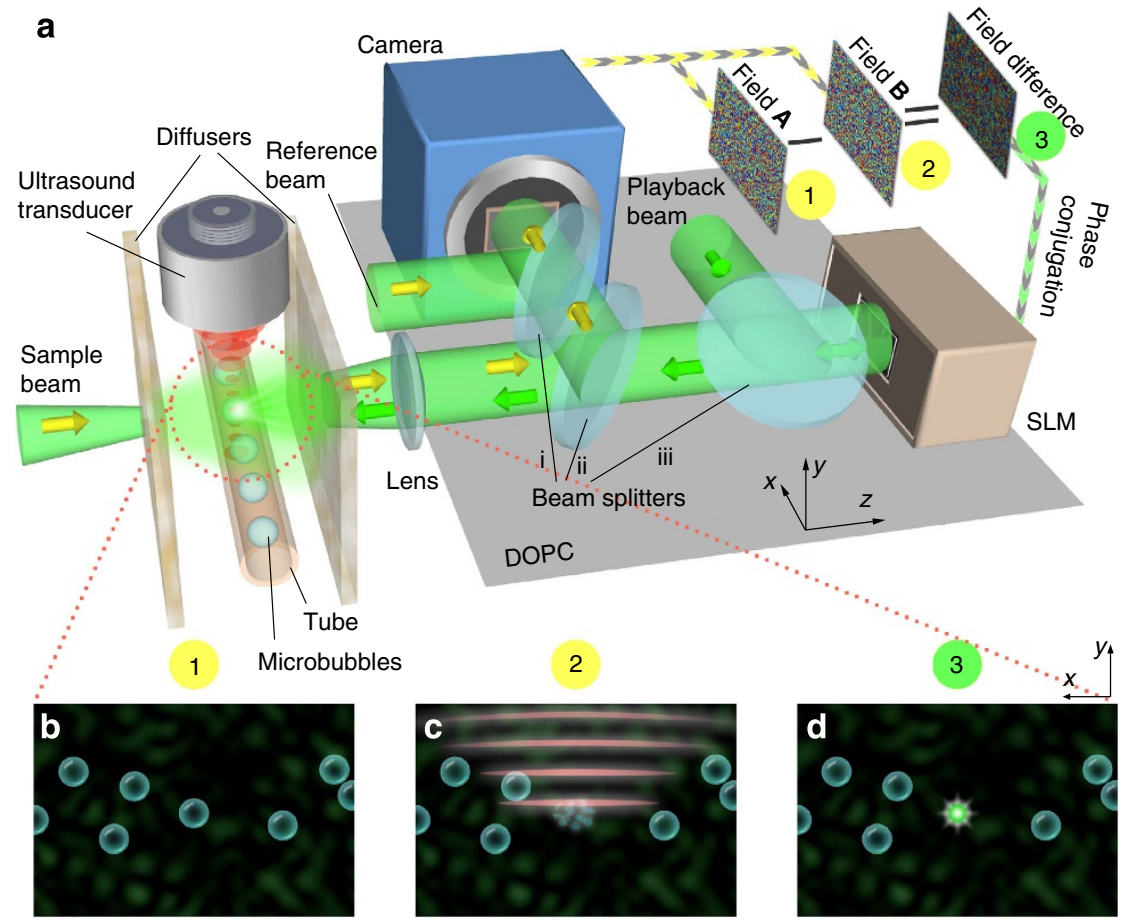

Figure 1 | Principle of TRUME technique. (a) Illustration of the experimental set-up (see Supplementary Figs 1 and 2 for schematic diagram in detail). The microbubbles perfuse inside an acrylic tube, which is sandwiched between two diffusers. A DOPC system is used as a phase conjugation mirror to timereverse the light back to the sample. (b-d) Illustration of TRUME optical focusing technique in three steps. At the first step, the camera of the DOPC system captures a transmitted optical field (Field $\mathbf{A}$ ) before applying ultrasound to the sample (b). Ultrasound bursts are then used to destroy the targeted microbubble (c), resulting in a different optical field (Field $\mathbf{B}$ ). The difference between two fields yields an optical field that appears to emerge from the destroyed microbubble. The conjugated phase of the difference field is then sent to the SLM to create a playback beam, which focuses light at the position of microbubble destruction (d). Yellow arrows, numbers and signal flows indicate recording process; green ones represent playback.

a

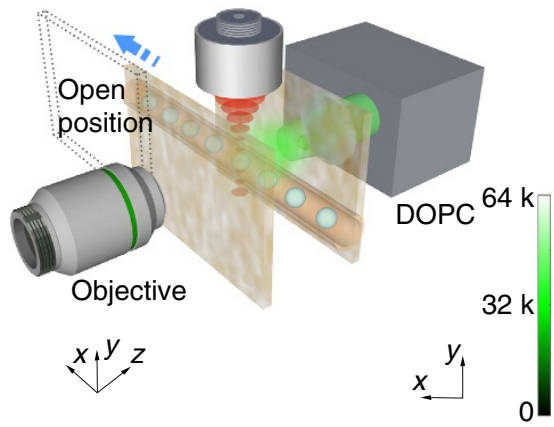

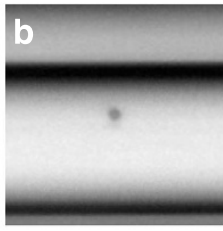
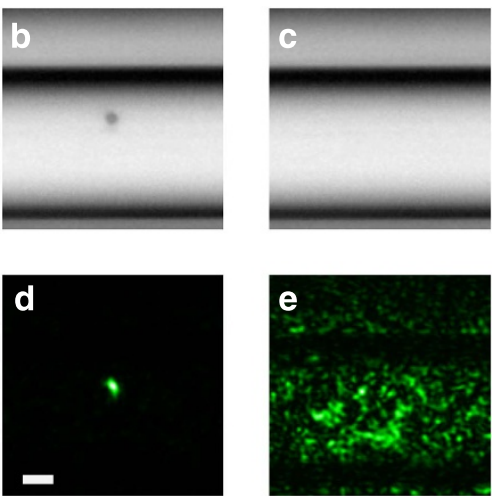

TRUME

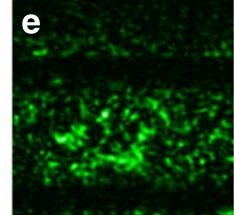

TRUE

Figure 2 | Visualization of the target plane. (a) Illustration of the observation set-up. The front diffuser was shifted to the open position before and after TRUME for direct visualization. A $\times 10$ microscope system was used to observe the target plane. (b,c) Images of a microbubble before and after applying ultrasound. (d) Optical focus created at the position of microbubble destruction. (e) Focusing results of TRUE technique. Scale bar, $10 \mu \mathrm{m}$. 
directly visualized the focus created at the position of destroyed microbubble (Fig. 2d). The measured peak intensity to background intensity ratio (PBR) of the TRUME focus in Fig. $2 \mathrm{~d}$ is $\sim 510$ (Supplementary Methods).

For comparison, we also measured the focusing profile of TRUE (Fig. 2e and Supplementary Methods). The PBR of the TRUME focus is around two orders of magnitude higher than that of TRUE (PBR $=\sim 2$ in Fig. 2e), since the TRUME concentrates light at fewer optical modes and has a stronger modulation efficiency per mode.

We separately measured the modulation efficiency of ultrasound in a clear medium (Supplementary Fig. 3 and Supplementary Methods), and found that $\sim 0.5 \%$ of light passing through the ultrasound focus ( $2 \mathrm{MPa}$ peak pressure) is modulated. In comparison, the proportion of light passing through the location of the bubble that is modulated by bubble destruction reaches $\sim 25 \%$ (Supplementary Fig. 3). This large difference in modulation efficiency is the primary reason why the TRUME guidestar offers a stronger focus.

Deep tissue optical focusing. To study the performance of TRUME for focusing through biological tissue, we used two pieces of 2-mm-thick biological tissue as our diffusive medium (see Methods section). The experimental set-up matches that shown in Fig. 2a. The images of the microbubble before and after destruction are shown in Fig. 3a,b. We directly observed the target plane (Fig. 3c-e) after the TRUME process. An optical focus (Fig. 3c,e) was created using TRUME, with PBR of $\sim 23$ (Supplementary Methods). Fitted Gaussian profiles (to the onedimensional data through the centre of the focus in the $x$ and $y$ directions) show the focus full width at half maximum (FWHM; Fig. $3 \mathrm{e}$ ) is $2.4 \pm 0.2 \mu \mathrm{m}$ in the $x$ direction and $1.7 \pm 0.2 \mu \mathrm{m}$ in the $y$ direction ( $95 \%$ confidence bounds). To confirm that this optical focus was created due to OPC, we shifted the SLM phase pattern in both $x$ and $y$ directions by 10 pixels. As shown in Fig. $3 \mathrm{~d}$, the optical focus vanishes as expected. The optical fields measured before and after microbubble destruction, as well as the subtracted field, are shown in Fig. $3 \mathrm{f}-\mathrm{h}$, respectively.

Demonstration of flow stream monitoring. One application of the TRUME focusing technique may be to perform cytometry behind a scattering media by using microbubbles, which are currently used as contrast agents in blood circulation ultrasound imaging ${ }^{34}$. To demonstrate this potential application (Fig. 4a), we mixed fluorescent microspheres $(4 \mu \mathrm{m})$ and microbubbles in $1 \mathrm{X}$ phosphate buffered saline and pumped the solution through an acrylic tube (see Methods section). We first formed an optical focus, as shown in Fig. $4 \mathrm{~b}$, by implementing the TRUME technique to focus on a microbubble at the target location. Fluorophores that subsequently flow across the focus then interact with the focused light spot to emit fluorescence. The fluorescence was filtered with an emission filter and detected by a single-photon-counting module outside the scattering medium (see Methods section). The resulting signal is shown in Fig. 4c. After counting, the front diffuser was shifted to the open position and the fluorescent microspheres were imaged with an emission filter for verification (Fig. 4d). The agreement of the results positively validates this proof-of-concept.

Addressable focus resolution improvement. Our demonstrations of TRUME thus far destroy an isolated microbubble with a relatively large ultrasound focus (one to two orders of magnitude larger), forming one sharp optical focus. If multiple microbubbles are clustered together, then the ultrasound focus may destroy more than one bubble. In this scenario, TRUME will generate an optical 'focus' significantly broader than the focus we have discussed thus far. To distinguish the two focus types, we will use the term addressable focus to refer to the achievable TRUME focus in the scenario where microbubbles are dense.

The addressable focus size is statistically determined by the pressure-to-destruction response of the bubbles. Interestingly, the probability of microbubble destruction varies nonlinearly as a function of pressure. In the ideal case where all microbubbles have the same destruction threshold, one can set the peak ultrasound pressure to be right at the threshold so that only the microbubble at the centre of the ultrasound focus will be destroyed and therefore obtain an addressable focus size that is equal to the single bubble TRUME focus size. In practice, however, the actual pressure-to-destruction response curve is not a simple step function. Nevertheless, the more nonlinear the response curve is, the sharper addressable focus we can achieve with TRUME.

To better characterize the pressure-to-destruction response and determine the TRUME addressable focus resolution achievable with our system, we experimentally measured the cumulative distribution function of the microbubble destruction $\sigma(P)$ by counting the number of microbubbles destroyed as a function of pressure (Supplementary Fig. 4 and Supplementary Methods). As
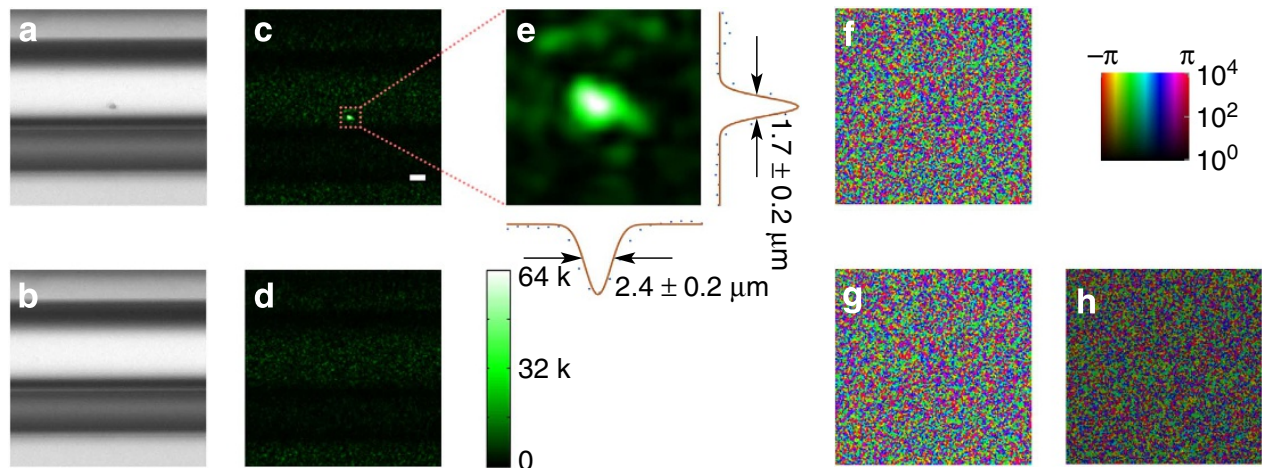

Figure 3 | Optical focusing in 2-mm-deep chicken tissue. Two pieces of 2-mm-thick chicken tissues were used as diffusers. (a) A microbubble in a tube before destruction. (b) After destruction. (c) A light focus was created at the position of the destroyed microbubble (PBR 23). (d) The optical focus vanished as the SLM shifts 10 pixels in both $x$ and $y$ directions. (e) 10X zoom-in image of the optical focus with quantified resolution. (f,g) Central part (200 pixels by 200 pixels) of the optical fields captured before (f) and after (g) the destruction of the microbubble. (h) Difference of the fields in $\mathbf{f}, \mathbf{g}$. Scale bar, $10 \mu \mathrm{m}$. 
shown in Fig. 5a (red), the cumulative distribution function reveals a strong nonlinear relationship between destruction probability and pressure. Given a focused ultrasound profile $P(x)$ (Fig. 5a, green; see also Methods section), we were able to calculate the microbubble destruction probability over position $\sigma(P(x))$ (Fig. 5a, blue), which predicts the addressable focus resolution of TRUME. The resulting profile is significantly narrower than the ultrasound pressure profile, implying that the nonlinear relationship would effectively improve the addressable focus resolution of TRUME.

To experimentally confirm the improvement of addressable focus resolution of TRUME, we used a thin microbubble sheet (see Methods section) to visualize the distribution of the foci as ultrasound pressure increases. To cover the entire ultrasound
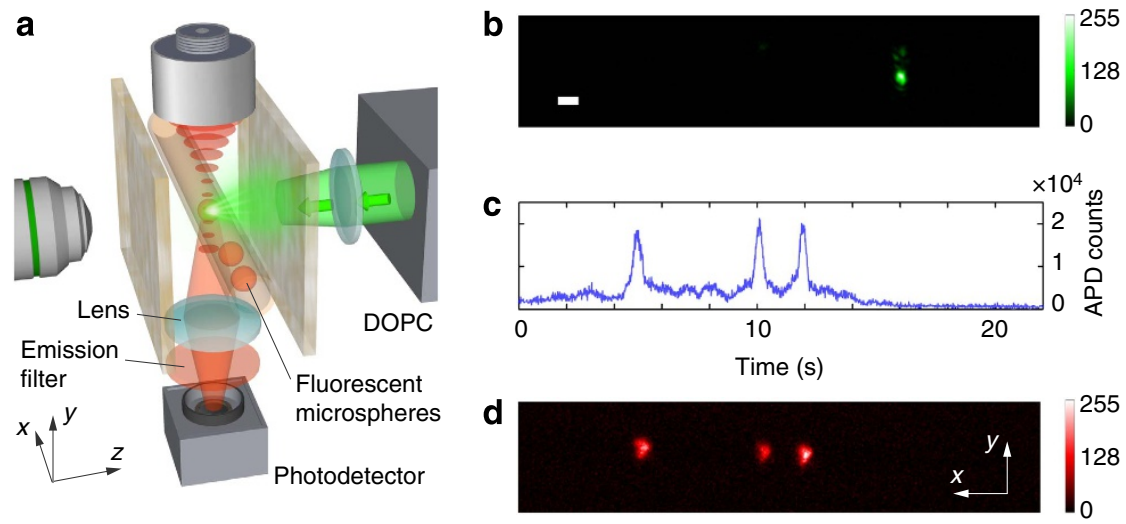

Figure 4 | Demonstration of flow stream monitoring through a scattering sample. (a) Illustration of the experimental set-up. An external single-photoncounting module (SPCM) was used to detect the excited fluorescence through the fluorescence filter. (b) A light focus was created with TRUME.

(c) Photon counts recorded by the SPCM as the optical focus probed the flowing microspheres. (d) Image of the fluorescent microspheres after passing through the optical focus in the $x$ direction. Scale bar, $10 \mu \mathrm{m}$.

a

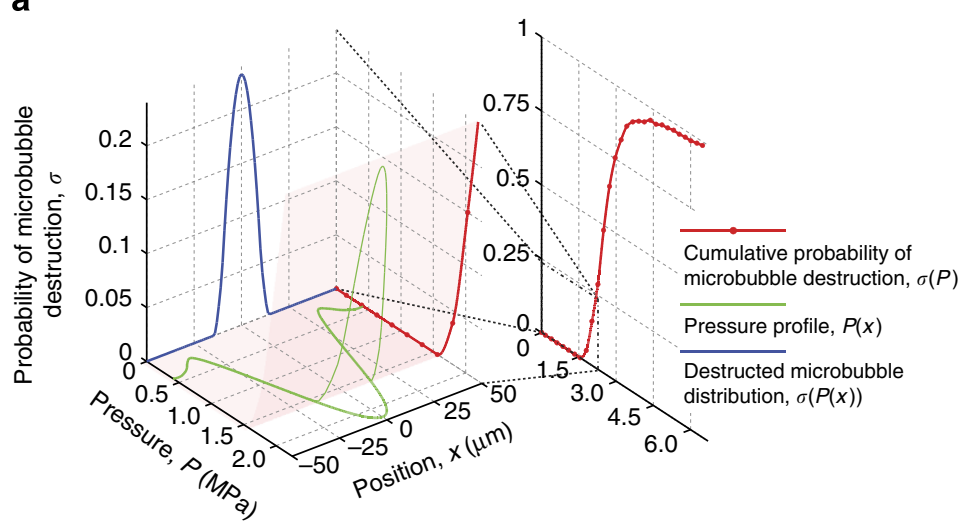

b

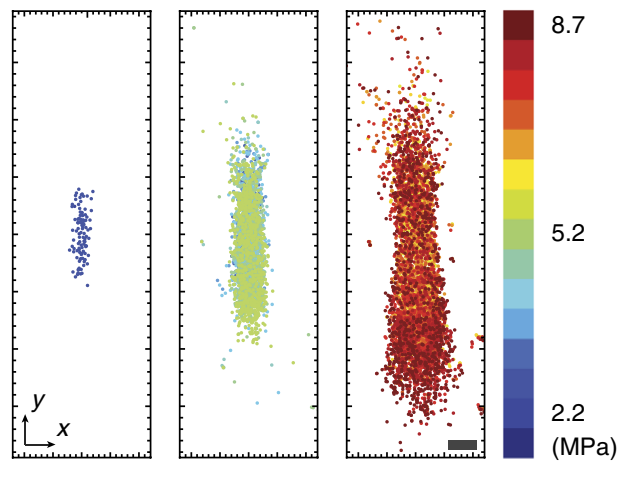

C

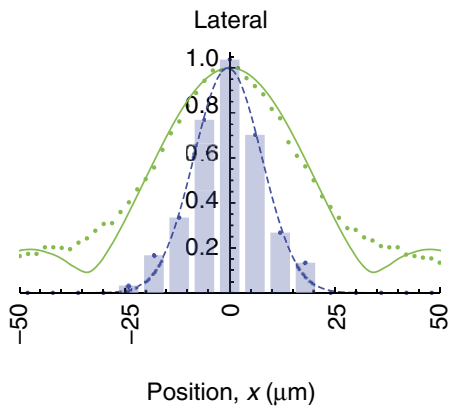

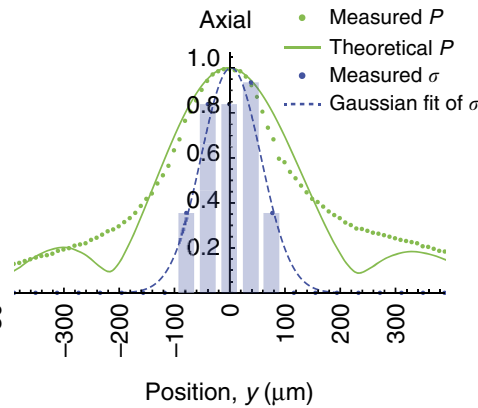

d

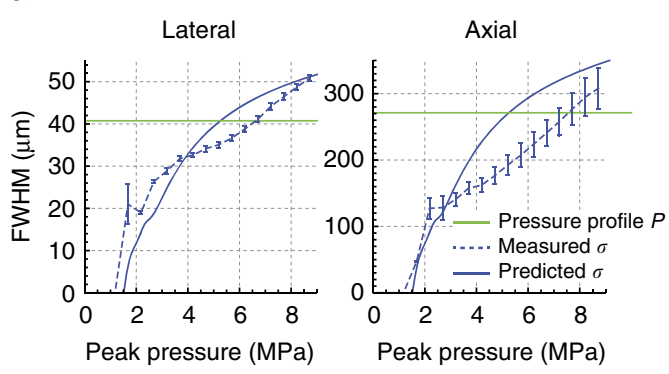

Figure 5 | Addressable focus resolution improvement by exploiting nonlinear microbubble destruction. (a) Calculation of microbubble destruction probability distribution over position (blue) based on the measured cumulative distribution function of the microbubble destruction $\sigma(P)$ (red) and the theoretical ultrasound pressure profile $P(x)$ (green). (b) Two-dimensional distribution map of TRUME foci over pressure levels. This distribution map aggregates centroids of the TRUME foci from 135 sets of data. (c) Comparison of the TRUME focus probability distribution (histograms, with Gaussian fit) and the ultrasound pressure profile (green) in both lateral (left) and axial (right) directions. The histograms were calculated from the low-pressure map (left figure in b). (d) Theoretical (for example, FWHM of the blue curve in $\mathbf{a}$ ) and experimental (for example, FWHM of the blue curve in c) TRUME addressable focus resolution over pressure. Green lines mark the FWHM of the ultrasound profile. Error bar indicates $95 \%$ confidence bounds. Scale bar in $\mathbf{b}, 50 \mu \mathrm{m}$. 
focus $(-6 \mathrm{~dB})$ with the current observation system and further improve the resolution, we used a $45-\mathrm{MHz}$, high numericaperture ultrasound beam with a measured beam diameter of $\sim 40 \mu \mathrm{m}$ and focal zone of $\sim 270 \mu \mathrm{m}$ ( $-6 \mathrm{~dB}$; see Methods section) in this experiment (see Supplementary Fig. 5 for the experimental results using $20 \mathrm{MHz}$ ultrasound). We applied 15 levels of ultrasound pressure (linearly from 1.7 to $8.7 \mathrm{MPa}$ ) to the sample and measured the fields before and after each insonation. We then played back the corresponding field difference sequentially, recorded the resulting focus patterns (Supplementary Fig. 6), and applied a watershed algorithm to extract each focus centroid (see Methods section). To collect meaningful statistics, this process was repeated 135 times at different unaffected regions of the microbubble sheet. We aggregated the measured TRUME focus centroids into a statistical map as shown in Fig. 5b, where foci are displayed in three pressure groups. The profile of the foci broadens as the ultrasound pressure becomes higher, confirming the nonlinearity effect in TRUME.

To quantify the addressable focus resolution improvement, we calculated the FWHM of Gaussian profiles that are fitted to the histograms of each statistical map along both lateral $(x)$ and axial $(y)$ directions. Figure $5 \mathrm{c}$ shows the Gaussian fits and histograms of the lower pressure group $(<2.2 \mathrm{MPa}$, Fig. $5 \mathrm{~b}$, left $)$ where microbubbles start to collapse. We also measured the ultrasound pressure profiles, which closely match with their theoretical profiles in both directions (see Methods section). The FWHM of the Gaussian fit to the centroid histogram in the lateral $(x)$ direction is $19 \mu \mathrm{m}$, while that of theoretical ultrasound focus is $40 \mu \mathrm{m}$. Likewise, the FWHM of the TRUME addressable focus along the axial $(y)$ direction is $130 \mu \mathrm{m}$, which is also lower than that of the ultrasound focus $(270 \mu \mathrm{m})$. We further studied the effect of ultrasound pressure on the nonlinearity-induced resolution improvement by calculating the FWHMs of the Gaussian fits of both the theoretical microbubble destruction distribution (for example, blue curve in Fig. 5a) and TRUME focus histogram profiles (for example, blue curve in Fig. 5c) at various pressure levels. As shown in Fig. 5d, both experimental and theoretical FWHMs are lower than that defined by the ultrasound focus (green line) when the ultrasound pressure is $<\sim 5 \mathrm{MPa}$. The discrepancy between these two curves is attributable to variations between the samples.

\section{Discussion}

Combining the advantages of a physical and a virtual guidestar, TRUME can selectively focus light to a size of $\sim 2 \mu \mathrm{m}$ in deep tissue, given the distribution of microbubbles it targets is sufficiently sparse. When the microbubble distribution is dense, we show that TRUME may still achieve an addressable focus resolution $\sim 2$ times higher than that defined by its ultrasound focus. As this method simply requires two measurements and no iterations, it is intrinsically fast and a good match with in vivo applications. Next, we list several factors that affect TRUME performance and outline several of its potential applications.

The size of an individual focus depends on that of the microbubble that is typically at micrometre scale, approximately tenfold smaller than a TRUE focus. Although ultrasound focus could cover multiple microbubbles, TRUME further confines the targeting range by taking the advantage of the nonlinear relationship between microbubble destruction probability and ultrasound pressure. The addressable focus resolution improvement was largely limited by the broad size distribution of the microbubbles, and thus can be enhanced by reducing the standard deviation of the radius of microbubbles, via separation techniques $^{44}$ or methods based on established protocols ${ }^{45,46}$.
Alternatively, simultaneously focusing to multiple microbubble locations might also be a desired experimental goal, like when using microbubbles as selective markers (for example, binding to certain disease markers) ${ }^{34}$.

The PBR of TRUME is measured to be two orders of magnitude greater than that of TRUE ( $\sim 510$ versus $\sim 2$, using a ground glass diffuser sample and the set-up in Fig. 2). Two factors lead to this large PBR increase. First, TRUME practically encodes significantly fewer optical modes, even if multiple microbubbles are present within the ultrasound focus. Second, the modulation efficiency of TRUME is much higher than TRUE. In our experiment, we found $\sim 25 \%$ modulation of the light passing through the TRUME guidestar. In comparison, a TRUE guidestar with a peak pressure of $2 \mathrm{MPa}$ only modulates $\sim 0.5 \%$ of its contained light.

The time needed to destroy a microbubble depends on the mechanisms of microbubble destruction, which can be classified into fragmentation and diffusion ${ }^{47}$. Fragmentation occurs when ultrasound pressure is relatively high and the microbubble is destroyed on a timescale of microseconds, which suggests the TRUME mechanism is likely applicable to in vivo tissue experiments. In the case where low ultrasound pressure is used, acoustic driven diffusion is the dominant destruction mechanism. This process typically spans tens of microseconds, depending on both the ultrasound parameters (pressure, frequency, cycles and so on) and microbubbles properties (size, shell material and encapsulated gas $)^{47}$. In this paper, the ultrasound pulse duration was $28.6 \mathrm{~ms}$ (one camera frame period), within which incomplete gas dissolution was also observed under certain circumstances, such as with low ultrasound pressure and a large microbubble diameter (Supplementary Movies 1-3). This effect results in a size decrease rather than complete disappearance of the microbubble. Intriguingly, decreasing the size of the microbubble between capturing two optical fields also enables TRUME to form an optical focus at the targeted microbubble (Supplementary Fig. 7 and Supplementary Note 1), because it shares the same effect as the complete microbubble destruction-inducing difference between two optical fields.

It should be noted that the lifetime of a TRUME focus depends on the tissue decorrelation time. To achieve a longer focusing duration, one may need to use the incomplete destruction approach. In this case, the TRUME focus can be repeatedly created by decreasing the size of the microbubble each time until complete destruction. Alternatively it is also possible to dynamically maintain the optical focus by using the ultrasounddriven microbubble oscillation effect ${ }^{48}$, which is also able to induce optical field variation.

Taking advantage of parallel field measurement, this DOPCbased technique creates optical foci in hundreds of milliseconds ( $\sim 280 \mathrm{~ms}$ in our experiments), a timescale short enough for ex vivo or even some in vivo biological applications with appropriate tissue immobilization methods ${ }^{49}$. It should be noted that no frame averaging was needed for any of our TRUME experiments. Like other field subtraction approaches, the background fields need to remain highly correlated as the light intensity fraction encoded by the microbubble is typically very small. This requirement suggests that we must capture the two fields in a sufficiently short time period to overcome in vivo tissue decorrelation given that microbubbles can induce field variation during this time interval. Technically, this can be achieved by using a high speed camera. Off-axis holography-based field measurement or binary phase measurement would further improve the system speed by reducing the number of frames needed for field measurement ${ }^{11,50}$.

Microbubbles are usually made with albumin or lipid, which stabilizes high molecular weight gases, such as perflutren. 
Microbubbles like these have been widely used as ultrasound contrast agents and proven for some applications in the human body. Their biocompatibility makes them a promising optical guidestar in biological tissue. Besides ultrasonic imaging, microbubbles also have promising applications in gene and drug delivery $^{51}$, where their ultrasonic destruction can release a therapeutic payload. Furthermore, microbubbles can also be targeted to regions of disease by surface conjugation of specific ligands or antibodies, which bind to the disease markers ${ }^{34}$. Recently, genetically encoded gas nanostructures from microorganisms have been demonstrated as a promising candidate as molecular reporters ${ }^{52}$. All these applications imply that microbubbles have high specificity and selectivity, with which TRUME focusing may provide precise optical mediation for drugs, cells or molecules. Example applications range from selective photo-thermal therapy for targeting tumour cells ${ }^{53,54}$ to specific light delivery in optogenetics ${ }^{55}$.

The prospect of using TRUME to perform imaging is less obvious and deserves some elaboration. Like other physical guidestar assisted wavefront shaping techniques, the TRUME focus position is collocated with the physical guidestar location and cannot be freely repositioned to perform raster scans. TRUME may potentially be combined with a newly described optical memory effect ${ }^{56}$ to perform scanning and imaging. Specifically, it has been demonstrated that lateral translation of the input optical wavefront can retain some of its focusing ability through scattering media with high scattering anisotropy. TRUME can potentially be used to generate the initial focus that can then be freely scanned within a small proximal region using this optical memory effect.

\section{Methods}

Set-up. The TRUME experiment was carried out in a custom-built set-up. The schematic diagram of the set-up is illustrated in Supplementary Fig. 1. A pulsed laser beam (532 nm wavelength, $7 \mathrm{~ns}$ pulse width, $20 \mathrm{kHz}$ repetition rate and $7 \mathrm{~mm}$ coherent length) generated from a Q-switch laser (Navigator, Spectra-Physics) was spilt into three beams: a sample beam, a reference beam and a playback beam. Both of the sample beam and the reference beam were shifted by $50 \mathrm{MHz}$ using an acousto-optical modulator (AFM-502-A1, IntraAction). The interference between the transmitted sample beam and reference beam was measured by the camera (PCO.edge, PCO) of the DOPC system. The playback beam was modulated with the conjugated phase of the subtracted field by an SLM (Pluto, Holoeye), which was precisely aligned to the camera through a beam splitter.

The $20-\mathrm{MHz}$ ultrasound burst was generated by a transducer with a $12.7-\mathrm{mm}$ focal length and $6.35 \mathrm{~mm}$ element diameter (V317, Olympus), and the $45-\mathrm{MHz}$ ultrasound burst was generated by a transducer that has a $6-\mathrm{mm}$ focal length and $6.35 \mathrm{~mm}$ element diameter (nominal frequency at $50 \mathrm{MHz}$, calibrated peak frequency at $44.4 \mathrm{MHz}, \mathrm{V} 3330$, Olympus). Both transducers were driven by a RF power amplifier (30W1000B, Amplifier Research).

To directly visualize the results, a custom-built microscope with a $\times 20$ objective (SLMPlan N, Olympus) and a tube lens of $100 \mathrm{~mm}$ focal length was used to image the target plane to a charge-coupled device camera (Stingray F145, Allied Vision Technologies). To demonstrate the cytometry application, the fluorescent signals were filtered by a 561-nm long-pass (LP02-561RE-25, Semrock) and a $582 / 75-\mathrm{nm}$ band-pass filter (FF01-582/75-25, Semrock) and detected by a single-photon-counting module (SPCM-AQRH-14, Perkinelmer).

Signal flow. The signal flow is shown in detail in Supplementary Fig. 2. The sample beam and reference beam were modulated by $50 \mathrm{MHz}$ signals generated from two channels of a function generator (AFG 3252, Tektronix). The optical field transmitted through the sample was measured by the camera (exposure time: $20 \mathrm{~ms}$, framerate: $35 \mathrm{fps}$ ) of the DOPC system using 4-phase shifting-based digital holography ${ }^{43}$. The phase shifting was synchronised with the camera exposure by controlling signals from a data acquisition card (PCI-6281, NI). The ultrasound burst signal ( 10 cycles, $10 \mu$ s interval) was generated by another function generator (4065, BK Precision) and time-gated $(28.6 \mathrm{~ms})$ by the data acquisition card.

Sample preparation. The microbubbles (Optison, GE health care) was diluted to $10 \%(\mathrm{v} / \mathrm{v} \%)$ in $1 \%(\mathrm{w} / \mathrm{w} \%)$ agarose gel in aqueous phase or $1 \mathrm{X}$ phosphate buffered saline (demonstration of flow stream monitoring) and perfused in an acrylic capillary tube (inner diameter: $50 \mu \mathrm{m}$, outer diameter: $100 \mu \mathrm{m}$, Paradigm Optics), which was positioned inside a clear polystyrene cuvette. Polyacrylamide gel (10\%) was used to fill the space in the cuvette to secure the capillary tube. Two diffusers $(10 \times 10 \mathrm{~mm}, 220$ grit ground glass, Edmund Optics) were placed outside the cuvette in parallel with $\sim 10 \mathrm{~mm}$ distance in between. The microbubble sheet was $\sim 20 \mu \mathrm{m}$ thick and sandwiched between two blocks of agarose gel with dimensions of $10 \mathrm{~mm}(x) \times 10 \mathrm{~mm}(y) \times 3 \mathrm{~mm}(z)$. The microbubble sheet was positioned between and parallel to the diffusers. The ultrasound beam was aligned to the microbubble sheet by maximizing the amplitude of the echo received from the focus.

In the flow stream monitoring experiment, fluorescent microspheres with $4 \mu \mathrm{m}$ diameter (FluoSpheres 580/605, Life Science) were used as targets. In the ex vivo tissue experiment, fresh chicken breast tissue was used as diffusive medium. For each tissue diffuser, a piece of 2 -mm-thick chicken breast tissue slice $(10 \mathrm{~mm}$ $(x) \times 10 \mathrm{~mm}(y))$ was sandwiched between two pieces of cover glass separated by a 2-mm spacer.

Ultrasound beam characterization. We calculated the theoretical ultrasound pressure field using the fast near-field method ${ }^{57}$. We first calculated the pressure fields at different single frequencies ranging from 1 to $100 \mathrm{MHz}$, and summed the profiles with a weight accounting for transducer response and frequency spectrum of ultrasound pulse train.

The ultrasound pressure was measured in room temperature water using a calibrated hydrophone (HGL-0085, Onda). To characterize the profile of the ultrasound beam, we operated the transducer in pulse-echo mode using a pulserreceiver (5900PR, Olympus) and scanned a line target (air filled polycarbonate tube, inner diameter $22.5 \mu \mathrm{m}$, outer diameter $25 \mu \mathrm{m}$, Paradigm Optics) by translating the transducer in the lateral and axial direction, respectively ${ }^{58}$. This method provides a more accurate measurement than using the hydrophone because the active diameter of the hydrophone is larger than the waist of the ultrasound beam generated by the V3330 transducer. The peak-peak voltages of the echoes were measured by an oscilloscope (DPO 3012, Tektronix). Because the measurement was based on single cycle burst, side lobes were not shown.

Watershed algorithm. We first binarized the image with a threshold that was seven times higher than the background intensity. This step outputs a binary image in which only the pixels around the peak have the value of 1 . We then segmented the binary image with a watershed algorithm and extracted the centroid of each focal spots.

\section{References}

1. Mosk, A. P., Lagendijk, A., Lerosey, G. \& Fink, M. Controlling waves in space and time for imaging and focusing in complex media. Nat. Photon. 6, 283-292 (2012).

2. Vellekoop, I. M. Feedback-based wavefront shaping. Opt. Express 23, 12189-12206 (2015)

3. Kim, M., Choi, W., Choi, Y., Yoon, C. \& Choi, W. Transmission matrix of a scattering medium and its applications in biophotonics. Opt. Express 23, 12648-12668 (2015).

4. Yu, H. et al. Recent advances in wavefront shaping techniques for biomedical applications. Curr. Appl. Phys. 15, 632-641 (2015).

5. Horstmeyer, R., Ruan, H. \& Yang, C. Guidestar-assisted wavefront-shaping methods for focusing light into biological tissue. Nat. Photon. 9, 563-571 (2015).

6. Vellekoop, I. M. \& Mosk, A. P. Focusing coherent light through opaque strongly scattering media. Opt. Lett. 32, 2309-2311 (2007).

7. Yaqoob, Z., Psaltis, D., Feld, M. S. \& Yang, C. Optical phase conjugation for turbidity suppression in biological samples. Nat. Photon. 2, 110-115 (2008).

8. Cui, M. \& Yang, C. Implementation of a digital optical phase conjugation system and its application to study the robustness of turbidity suppression by phase conjugation. Opt. Express 18, 3444-3455 (2010).

9. Papadopoulos, I. N., Farahi, S., Moser, C. \& Psaltis, D. Focusing and scanning light through a multimode optical fiber using digital phase conjugation. Opt. Express 20, 10583-10590 (2012).

10. Hillman, T. R. et al. Digital optical phase conjugation for delivering twodimensional images through turbid media. Sci. Rep. 3, 1909 (2013)

11. Wang, D. et al. Focusing through dynamic tissue with millisecond digital optical phase conjugation. Optica 2, 728-735 (2015).

12. Lee, K., Lee, J., Park, J.-H., Park, J.-H. \& Park, Y. One-wave optical phase conjugation mirror by actively coupling arbitrary light fields into a single-mode reflector. Phys. Rev. Lett. 115, 153902 (2015).

13. Popoff, S. M. et al. Measuring the transmission matrix in optics: an approach to the study and control of light propagation in disordered media. Phys. Rev. Lett. 104, 100601 (2010).

14. Yu, H. et al. Measuring large optical transmission matrices of disordered media. Phys. Rev. Lett. 111, 153902 (2013).

15. Chaigne, T. et al. Controlling light in scattering media non-invasively using the photoacoustic transmission matrix. Nat. Photon. 8, 58-64 (2013).

16. Hsieh, C., Pu, Y., Grange, R. \& Psaltis, D. Digital phase conjugation of second harmonic radiation emitted by nanoparticles in turbid media. Opt. Express 18, 533-537 (2010). 
17. Vellekoop, I. M., Cui, M. \& Yang, C. Digital optical phase conjugation of fluorescence in turbid tissue. Appl. Phys. Lett. 101, 081108 (2012).

18. Katz, O., Small, E., Guan, Y. \& Silberberg, Y. Noninvasive nonlinear focusing and imaging through strongly scattering turbid layers. Optica 1, 170-174 (2014).

19. Zhou, E. H., Ruan, H., Yang, C. \& Judkewitz, B. Focusing on moving targets through scattering samples. Optica 1, 227-232 (2014).

20. Ma, C., Xu, X., Liu, Y. \& Wang, L. V. Time-reversed adapted-perturbation (TRAP) optical focusing onto dynamic objects inside scattering media. Nat. Photon. 8, 931-936 (2014).

21. Kong, F. et al. Photoacoustic-guided convergence of light through optically diffusive media. Opt. Lett. 36, 2053-2055 (2011).

22. Caravaca-Aguirre, A. M. et al. High contrast three-dimensional photoacoustic imaging through scattering media by localized optical fluence enhancement. Opt. Express 21, 26671-26676 (2013).

23. Lai, P., Wang, L., Tay, J. W. \& Wang, L. V. Photoacoustically guided wavefront shaping for enhanced optical focusing in scattering media. Nat. Photon. 9, 126-132 (2015).

24. Xu, X., Liu, H. \& Wang, L. V. Time-reversed ultrasonically encoded optical focusing into scattering media. Nat. Photon. 5, 154-157 (2011).

25. Wang, Y. M., Judkewitz, B., DiMarzio, C. A. \& Yang, C. Deep-tissue focal fluorescence imaging with digitally time-reversed ultrasound-encoded light. Nat. Commun. 3, 928 (2012).

26. Si, K., Fiolka, R. \& Cui, M. Fluorescence imaging beyond the ballistic regime by ultrasound pulse guided digital phase conjugation. Nat. Photon. 6, 657-661 (2012).

27. Ma, C., Xu, X. \& Wang, L. V. Analog time-reversed ultrasonically encoded light focusing inside scattering media with a 33,000 $\times$ optical power gain. Sci. Rep. 5, 8896 (2015).

28. Jang, M., Ruan, H., Judkewitz, B. \& Yang, C. Model for estimating the penetration depth limit of the time-reversed ultrasonically encoded optical focusing technique. Opt. Express 22, 5787-5807 (2014).

29. Kothapalli, S.-R. \& Wang, L. V. Ultrasound-modulated optical microscopy. J. Biomed. Opt. 13, 054046 (2008).

30. Si, K., Fiolka, R. \& Cui, M. Breaking the spatial resolution barrier via iterative sound-light interaction in deep tissue microscopy. Sci. Rep. 2, 748 (2012).

31. Ruan, H., Jang, M., Judkewitz, B. \& Yang, C. Iterative time-reversed ultrasonically encoded light focusing in backscattering mode. Sci. Rep. 4, 7156 (2014).

32. Suzuki, Y., Tay, J. W., Yang, Q. \& Wang, L. V. Continuous scanning of a timereversed ultrasonically encoded optical focus by reflection-mode digital phase conjugation. Opt. Lett. 39, 3441-3444 (2014).

33. Judkewitz, B., Wang, Y. M., Horstmeyer, R., Mathy, A. \& Yang, C. Speckle-scale focusing in the diffusive regime with time-reversal of variance-encoded light (TROVE). Nat. Photon. 7, 300-305 (2013).

34. Lindner, J. R. Microbubbles in medical imaging: current applications and future directions. Nat. Rev. Drug Discov. 3, 527-532 (2004).

35. Goertz, D. E. et al. High frequency nonlinear B-scan imaging of microbubble contrast agents. IEEE Trans. Ultrason. Ferroelectr. Freq. Control 52, 65-79 (2005).

36. Viessmann, O. M., Eckersley, R. J., Christensen-Jeffries, K., Tang, M. X. \& Dunsby, C. Acoustic super-resolution with ultrasound and microbubbles. Phys. Med. Biol. 58, 6447-6458 (2013).

37. O’Reilly, M. A. \& Hynynen, K. A super-resolution ultrasound method for brain vascular mapping. Med. Phys. 40, 110701 (2013).

38. Desailly, Y., Couture, O., Fink, M. \& Tanter, M. Sono-activated ultrasound localization microscopy. Appl. Phys. Lett. 103, 174107 (2013).

39. Benchimol, M. J. et al. Phospholipid/Carbocyanine Dye-Shelled Microbubbles as Ultrasound-Modulated Fluorescent Contrast Agents. Soft Matter 9, 2384-2388 (2013).

40. Liu, Y., Feshitan, J. A., Wei, M.-Y., Borden, M. A. \& Yuan, B. Ultrasoundmodulated fluorescence based on fluorescent microbubbles. J. Biomed. Opt. 19, 085005 (2014).

41. Ruan, H., Mather, M. L. \& Morgan, S. P. Ultrasound modulated optical tomography contrast enhancement with non-linear oscillation of microbubbles. Quant. Imaging Med. Surg. 5, 9-16 (2015).

42. Jang, M., Ruan, H., Zhou, H., Judkewitz, B. \& Yang, C. Method for autoalignment of digital optical phase conjugation systems based on digital propagation. Opt. Express 22, 14054-14071 (2014).

43. Yamaguchi, I., Matsumura, T. \& Kato, J.-I. Phase-shifting color digital holography. Opt. Lett. 27, 1108-1110 (2002).

44. Shekhar, H., Rychak, J. J. \& Doyley, M. M. Modifying the size distribution of microbubble contrast agents for high-frequency subharmonic imaging. Med. Phys. 40, 082903 (2013).
45. Pancholi, K. P., Farook, U., Moaleji, R., Stride, E. \& Edirisinghe, M. J. Novel methods for preparing phospholipid coated microbubbles. Eur. Biophys. J. 37, 515-520 (2008).

46. Palanchon, P., Klein, J. \& de Jong, N. Production of standardized air bubbles: application to embolism studies. Rev. Sci. Instrum. 74, 2558-2563 (2003).

47. Chomas, J. E., Dayton, P., Allen, J., Morgan, K. \& Ferrara, K. W. Mechanisms of contrast agent destruction. IEEE Trans. Ultrason. Ferroelectr. Freq. Control 48, 232-248 (2001).

48. Thomas, D. H., Sboros, V., Emmer, M., Vos, H. \& de Jong, N. Microbubble oscillations in capillary tubes. IEEE Trans. Ultrason. Ferroelectr. Freq. Control 60, 105-114 (2013).

49. Jang, M. et al. Relation between speckle decorrelation and optical phase conjugation (OPC)-based turbidity suppression through dynamic scattering media: a study on in vivo mouse skin. Biomed. Opt. Express 6, 72-85 (2015).

50. Ma, C., Zhou, F., Liu, Y. \& Wang, L. V. Single-exposure optical focusing inside scattering media using binarized time-reversed adapted perturbation. Optica 2, 869 (2015).

51. Ferrara, K., Pollard, R. \& Borden, M. Ultrasound microbubble contrast agents fundamentals and application to gene and drug delivery. Annu. Rev. Biomed. Eng. 9, 415-447 (2007).

52. Shapiro, M. G. et al. Biogenic gas nanostructures as ultrasonic molecular reporters. Nat. Nanotechnol. 9, 311-316 (2014).

53. El-Sayed, I. H., Huang, X. \& El-Sayed, M. A. Selective laser photo-thermal therapy of epithelial carcinoma using anti-EGFR antibody conjugated gold nanoparticles. Cancer Lett. 239, 129-135 (2006).

54. Schnarr, K. et al. Gold nanoparticle-loaded neural stem cells for photothermal ablation of cancer. Adv. Healthc. Mater. 2, 976-982 (2013).

55. Gradinaru, V., Mogri, M., Thompson, K. R., Henderson, J. M. \& Deisseroth, K. Optical deconstruction of parkinsonian neural circuitry. Science 324, 354-359 (2009).

56. Judkewitz, B., Horstmeyer, R., Vellekoop, I. M., Papadopoulos, I. N. \& Yang, C. Translation correlations in anisotropically scattering media. Nat. Phys. 11, 684-689 (2015).

57. Chen, D. \& McGough, R. J. A 2D fast near-field method for calculating nearfield pressures generated by apodized rectangular pistons. J. Acoust. Soc. Am. 124, 1526-1537 (2008).

58. Raum, K. \& O’Brien, W. D. Pulse-echo field distribution measurement technique for high-frequency ultrasound sources. IEEE Trans. Ultrason. Ferroelectr. Freq. Control 44, 810-815 (1997).

\section{Acknowledgements}

We thank Dr Daifa Wang for developing the idea, Mr Roarke Horstmeyer for the comments on the mathematics and the manuscript, and Mr Joshua Brake as well as $\mathrm{Mr}$ Edward Zhou for helpful discussions. We also like to thank Dr Mikhail G. Shapiro, Dr Stephen P. Morgan and Dr Melissa L. Mather for the discussions on microbubbles. This work is supported by the National Institutes of Health (1DP2OD007307-01), the National Institutes of Health BRAIN Initiative (1U01NS090577-01) and the GIST-Caltech Collaborative Research Proposal (CG2012).

\section{Author contributions}

H.R. and M.J. contributed equally to the work. H.R. conceived the idea. H.R., M.J. and C.Y. developed the idea and designed the experiments, which were carried out by H.R. and M.J. Data analysis was carried out by H.R. and M.J. with help from C.Y. who also supervised the project. All authors contributed to the preparation of the manuscript.

\section{Additional information}

Supplementary Information accompanies this paper at http://www.nature.com/ naturecommunications

Competing financial interests: The authors declare no competing financial interests.

Reprints and permission information is available online at http://npg.nature.com/ reprintsandpermissions/

How to cite this article: Ruan, H. et al. Optical focusing inside scattering media with time-reversed ultrasound microbubble encoded light. Nat. Commun. 6:8968 doi: 10.1038/ncomms9968 (2015).

This work is licensed under a Creative Commons Attribution 4.0 International License. The images or other third party material in this article are included in the article's Creative Commons license, unless indicated otherwise in the credit line; if the material is not included under the Creative Commons license, users will need to obtain permission from the license holder to reproduce the material. To view a copy of this license, visit http://creativecommons.org/licenses/by/4.0/ 